\title{
An Analytical Calculation of the Magnetic Field Using the Biot Savart Law
}

Um Cálculo Analítico do Campo Magnético Usando a Lei de Biot Savart

\author{
E.C. Caparelli and D. Tomasi \\ Escuela de Ciencia y Tecnologia, Universidad Nacional de Gral. \\ San Martin, Alem 3901, 1651, San Andres, Buenos Aires, Argentina
}

Recebido em 20 de Junho 2001. Aceito em 6 de Agosto 2001.

\begin{abstract}
This work presents an analytical method to calculate the magnetic field at any point of the space, by solving the Biot Savart equation in the reciprocal space. This is applied to express the magnetic field due to a circular current distributions as a convergent series. The comparison between the proposed method with the standard numerical integration of the Biot Savart law has shown a good agreement.
\end{abstract}

Neste trabalho apresentamos um método analítico para calcular o campo magnético em qualquer ponto do espaço, resolvendo a equação de Biot Savart no espaço recíproco. O mesmo é aplicado para expressar em termos de uma série convergente o campo magético gerado por uma distribuição de corrente circular. A comparação entre o método proposto e a integração numérica usual da lei de Biot Savart mostra uma satisfatória concordância.

\section{Introduction}

The Biot Savart (BS) law is a well known equation used to calculate the magnetic field vector at any point of the space for a given current distribution. Although it has extensive application in many branches of physics and engineering, analytical solutions of this integral equation are available only for some current distributions, which have specials symmetry conditions.

Usually numerical methods are used to calculate magnetic fields, for example, to design coils and magnets that produce high homogeneous magnetic fields for Magnetic Resonance Imaging(MRI) [1], to optimize toroidal magnetic systems [2] or to evaluate the stress in fusion devices [3]. However, when the calculation of the magnetic field is performed over a large set of points, the increase in the computing time requires the use of semi-analytic approaches [4].

In this work we are showing that the BS law can be solved in the reciprocal space for a planar current distribution. As an example, we derived analytically an expression to calculate the magnetic field at any point of the space for a circular turn of wire carrying a current.

\section{The method}

The magnetic field vector $\mathbf{B}$ at a given point in the space, $\mathbf{r}$, is related to the current distribution $\mathbf{j}\left(\mathbf{r}^{\prime}\right)$ through the Biot Savart law [5]

$$
\mathbf{B}(\mathbf{r})=\frac{\mu_{0}}{4 \pi} \int \frac{\mathbf{j}\left(\mathbf{r}^{\prime}\right) \times\left|\mathbf{r}-\mathbf{r}^{\prime}\right|}{\left|\mathbf{r}-\mathbf{r}^{\prime}\right|^{3}} d^{3} r^{\prime},
$$

where $\mu_{0}$ is the permeability of the free space and the integral is performed over the whole space. This law can also be expressed as a function of the curl of the vector potential, $\mathbf{A}(\mathbf{r})$, as

$$
\mathbf{B}(\mathbf{r})=\frac{\mu_{0}}{4 \pi} \nabla \times \int \frac{\mathbf{j}\left(\mathbf{r}^{\prime}\right)}{\left|\mathbf{r}-\mathbf{r}^{\prime}\right|} d^{3} r^{\prime}=\nabla \times \mathbf{A}(\mathbf{r}),
$$

that, using the Green function expansion [6]

$$
G\left(\mathbf{r}, \mathbf{r}^{\prime}\right)=\frac{1}{\left|\mathbf{r}-\mathbf{r}^{\prime}\right|}=\frac{1}{2 \pi^{2}} \int \frac{e^{i \mathbf{k} \cdot\left(\mathbf{r}-\mathbf{r}^{\prime}\right)}}{k^{2}} d^{3} k
$$

can be written as

$$
\mathbf{B}(\mathbf{r})=\frac{\mu_{0}}{8 \pi^{3}} \nabla \times \iint \mathbf{j}\left(\mathbf{r}^{\prime}\right) \frac{e^{i \mathbf{k} \cdot\left(\mathbf{r}-\mathbf{r}^{\prime}\right)}}{k^{2}} d^{3} k d^{3} r^{\prime} .
$$

Now, considering the Fourier transform of $\mathbf{j}\left(\mathbf{r}^{\prime}\right)$

$$
\mathbf{j}(\mathbf{k})=\int \mathbf{j}\left(\mathbf{r}^{\prime}\right) e^{-i \mathbf{k} \cdot \mathbf{r}^{\prime}} d^{3} r^{\prime}
$$


the curl can be carried out, and the Eq. (4) can be expressed as

$$
\mathbf{B}(\mathbf{r})=\frac{-i \mu_{0}}{8 \pi^{3}} \int \frac{\mathbf{j}(\mathbf{k}) \times \mathbf{k}}{k^{2}} e^{i \mathbf{k} \cdot \mathbf{r}} d^{3} k
$$

If the current distribution is in the $(x, y, 0)$ plane, the Fourier transform of this distribution can be written as

$$
\mathbf{j}(\mathbf{k})=j_{x}\left(k_{x}, k_{y}\right) \hat{\mathbf{i}}+j_{y}\left(k_{x}, k_{y}\right) \hat{\mathbf{j}}
$$

and the continuity equation, expressed in terms of the Fourier components, is given by [7]

$$
k_{x} j_{x}\left(k_{x}, k_{y}\right)+k_{y} j_{y}\left(k_{x}, k_{y}\right)=0 .
$$

Then the magnetic field components due to this current distribution can be expressed, using Eq. (6), as

$$
\begin{gathered}
B_{\alpha}(\mathbf{r})=\int k_{\alpha} k_{z} f(\mathbf{k}, \mathbf{r}) d^{3} k, \quad \alpha=x, y \\
B_{z}(\mathbf{r})=-\int\left(k_{x}^{2}+k_{y}^{2}\right) f(\mathbf{k}, \mathbf{r}) d^{3} k
\end{gathered}
$$

where

$$
f(\mathbf{k}, \mathbf{r})=\frac{i \mu_{0}}{8 \pi^{3}} \frac{j_{x}\left(k_{x}, k_{y}\right)}{k_{y}} \frac{e^{i \mathbf{k} \cdot \mathbf{r}}}{k^{2}} .
$$

Therefore, upon integrating over $k_{z}$, we finally have

$$
B_{\beta}(\mathbf{r})=\frac{i \mu_{0}}{8 \pi^{2}} \frac{\partial}{\partial \beta} \int_{\infty}^{\infty} \int_{\infty}^{\infty} \frac{e^{i\left(k_{x} x+k_{y} y\right)}}{k_{y}} j_{x}\left(k_{x}, k_{y}\right) e^{-|z| \sqrt{k_{x}^{2}+k_{y}^{2}}} d k_{x} d k_{y}, \quad \beta=x, y, z
$$

which are analytical expressions for the magnetic field components produced by a planar current distribution.

\section{Application}

Now we will consider a current distribution defined by a single loop of radius $R$, which carries a current $I$, as it is illustrated by Fig. 1. In this case the Fourier transform of the complex current density can be written as $[7]$

$$
j_{x}\left(k_{x}, k_{y}\right)+i j_{y}\left(k_{x}, k_{y}\right)=2 \pi I R e^{i \phi} J_{1}(R q),
$$

where $k_{x}+i k_{y}=q e^{i \phi}$ is a complex number with modulus $q=\sqrt{k_{x}^{2}+k_{y}^{2}}$ and $J_{1}(R q)$ is the Bessel function of order 1. Using the Eq. (8), the expression (13) can be reduced to

$$
j_{x}\left(k_{x}, k_{y}\right)=i 2 \pi I \frac{k_{y}}{q} R J_{1}(q R)
$$

The magnetic field components are then obtained substituting this current distribution in Eq. (12)

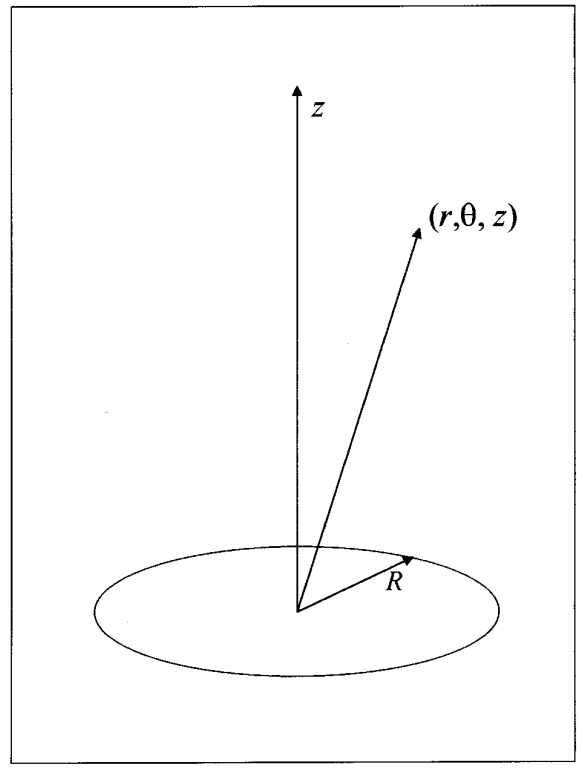

Figure 1: The circular current distribution diagram and the cylindric reference frame.

$$
B_{\beta}(\mathbf{r})=-\frac{\mu_{0} I R}{4 \pi} \frac{\partial}{\partial \beta} \int_{0}^{\infty} J_{1}(q R) e^{-|z| q} \int_{0}^{2 \pi} e^{i q(x \cos \phi+y \sin \phi)} d \phi d q,
$$

where the angular integral can be written as the convergent series [8]

$$
\int_{0}^{2 \pi} e^{i q(x \cos (\phi)+y \sin (\phi))} d \phi=2 \pi \sum_{k=0}^{\infty}(-1)^{k} \frac{(q r)^{2 k}}{\left(2^{k} k !\right)^{2}}, \quad r^{2}=x^{2}+y^{2} .
$$


Because of the axial symmetry of this problem we will use the cylindrical representation of the magnetic field vector. The radial component $B_{r}^{2}(\mathbf{r})=B_{x}^{2}(\mathbf{r})+B_{y}^{2}(\mathbf{r})$ and the axial component $B_{z}(\mathbf{r})$ of the magnetic field can be obtained from Eq. (15) and can be written as the series expansion

$$
B_{\gamma}(\mathbf{r})=\frac{\mu_{0} I R}{2} \frac{\partial}{\partial \gamma} \sum_{k=0}^{\infty}(-1)^{k+1} \frac{r^{2 k}}{\left(2^{k} k !\right)^{2}} \int_{0}^{\infty} q^{2 k} e^{-|z| q} J_{1}(q R) d q, \quad \gamma=r, z
$$

The integral over $q$ is related to the hypergeometric function, ${ }_{2} F_{1}$ as [8]

$$
\int_{0}^{\infty} q^{2 k} e^{-|z| q} J_{1}(q R) d q=\frac{R}{2}|z|^{2(k+1)} \Gamma[2(k+1)]_{2} F_{1}\left(k+1, k+\frac{3}{2}, 2 ;-\frac{R^{2}}{z^{2}}\right),
$$

which is defined as [8]

$$
\begin{gathered}
{ }_{2} F_{1}(a, b, c ; w)=\sum_{n=0}^{\infty} \frac{(a)_{n}(b)_{n}}{(c)_{n}} \frac{w^{n}}{n !} \\
(d)_{n}=\frac{\Gamma(d+n)}{\Gamma(d)} \quad \text { for } \quad d=a, b, c .
\end{gathered}
$$

Finally the magnetic field components can be written as the series

$$
\begin{gathered}
B_{r}(\mathbf{r})=\frac{\mu_{0} I R^{2}}{2} \sum_{k=0}^{\infty}(-1)^{k+1} \frac{k r^{2 k-1}}{\left(2^{k} k !\right)^{2}} z^{-2(k+1)} \Gamma[2(k+1)]_{2} F_{1}\left(k+1, k+\frac{3}{2}, 2 ;-\frac{R^{2}}{z^{2}}\right), \\
B_{z}(\mathbf{r})=\frac{\mu_{0} I R^{2}}{2} \sum_{k=0}^{\infty}(-1)^{k} \frac{(k+1) r^{2 k}}{\left(2^{k} k !\right)^{2}}|z|^{-(2 k+3)} \Gamma[2(k+1)]_{2} F_{1}\left(k+\frac{3}{2}, k+2,2 ;-\frac{R^{2}}{z^{2}}\right) .
\end{gathered}
$$

In table 1 , we show some expressions of the function ${ }_{2} F_{1}$.

Table 1: Values of Hypergeometric function ${ }_{2} F_{1}$

\begin{tabular}{|c|c|c|}
\hline$k=0$ & $2\left(\frac{z}{R}\right)^{2}\left(1-\left(1+\left(\frac{R}{z}\right)^{2}\right)^{-1 / 2}\right)$ & ${ }_{2} F_{1}\left(k+\frac{3}{2}, k+2,2 ;-\frac{R^{2}}{z^{2}}\right)$ \\
\hline$k=1$ & $\left(1+\left(\frac{R}{z}\right)^{2}\right)^{-5 / 2}$ & $\left(1+\left(\frac{R}{z}\right)^{2}\right)^{-3 / 2}$ \\
$k=2$ & $\left(1-\frac{3}{4}\left(\frac{R}{z}\right)^{2}\right)\left(1+\left(\frac{R}{z}\right)^{2}\right)^{-7 / 2}$ & $\left(1-\frac{1}{4}\left(\frac{R}{z}\right)^{2}\right)\left(1+\left(\frac{R}{z}\right)^{2}\right)^{-7 / 2}$ \\
\hline
\end{tabular}

Note that, in the Ref. [5], the magnetic field for a single loop is calculated analytically using the vector potential, whose solutions are valid only near the axis, near the center of the loop and far from the loop, but here we got analytical expressions that are valid at any region.

\section{Analysis}

To study the quality of the above result as a function of the number of terms $N$, considered in the Eqs. (21) and (22), we calculated the magnetic field generated by a current of $I=1 \mathrm{~A}$ flowing in a loop with $R=1 \mathrm{~m}$ in two ways: on one hand we used the MATHEMATICA $3.0^{1}$ software to carry out the operations involved in
Eqs. (21) and (22), and on the other hand we wrote a double precision FORTRAN code performing the integral in Eq. (1). In order to maximize the accuracy of the calculation accomplished by the numerical algorithm, we divided the wire in 8192 elements.

Figure 2 compares the $z$-component of the magnetic field obtained from Eq. (22), for different values of $N$, with that achieved by the numerical integration of Eq. (1) (solid line), along the radial direction at $z=0$. As can be noticed the increase in the number of terms in the series expansion (22) results in an improvement of the matching between the analytical solution and the numerical calculation of the BS law. Whereas for $r<0.8 R$ both calculations give similar results, for $r$

\footnotetext{
${ }^{1}$ MATHEMATICA is a registered trademark of Wolfram Research, Inc.
} 
approaching $R$ a mismatch between the two methods is observed. This mismatch corresponds to the cut off in the series expansion (22), while for $r>R$ this behavior is due to the non cenvergence of the series (22).

The difference between the analytical method, $B_{\gamma}^{(A n)}(r, z)$, and the standard BS method, $B_{\gamma}^{(B S)}(r, z)$, can be analyzed using the relative difference between the magnetic fields (in part per million - ppm) obtained from both methods,

$$
\Delta B_{\gamma}(r, z)=10^{6}\left|1-\frac{B_{\gamma}^{(A n)}(r, z)}{B_{\gamma}^{(B S)}(r, z)}\right|
$$

Figure 3 shows $\Delta B_{z}(r, z=0)$ as a function of the radial position in the central transverse plane. While for $N=5$ both methods differ less than $10 \mathrm{ppm}$ at $r \leq 0.4 R$, for $N=10$ the matching region is extended to $r \leq 0.6 R$, showing the importance of the number of terms in the $B_{z}(r, z)$ series.

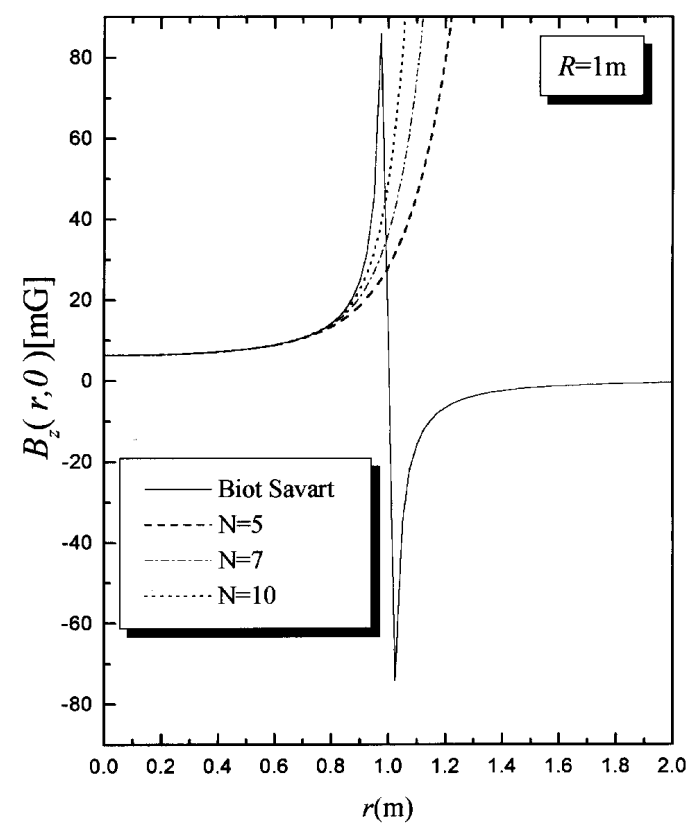

Figure 2: $B_{z}(r, z=0)$ calculated by the standard BiotSavart method and by the proposed analytical method for different values of $N$, as function of the radial position.

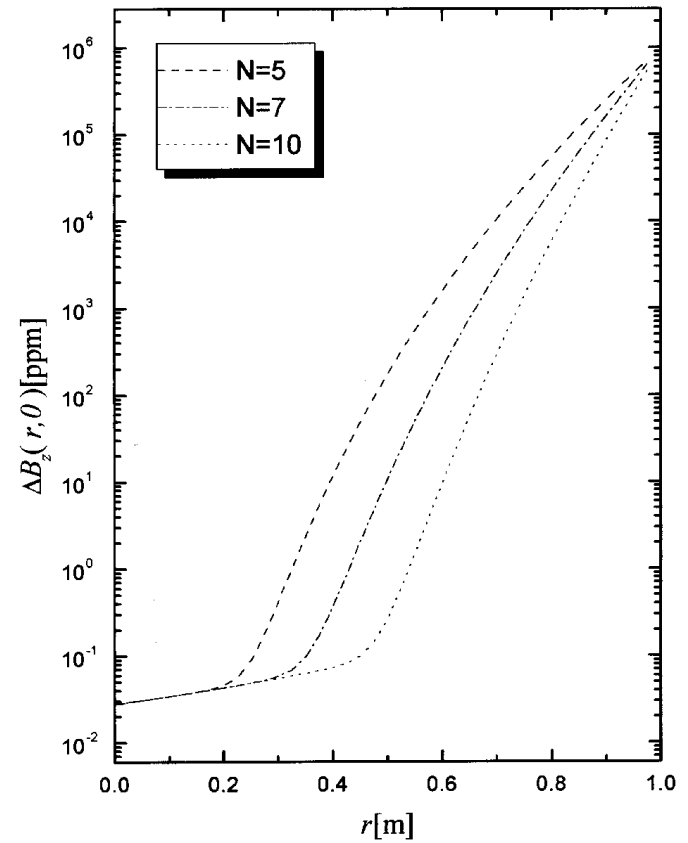

Figure 3: $\Delta B_{z}(r, z=0)$ as a function of the radial position.

(A)

(B)

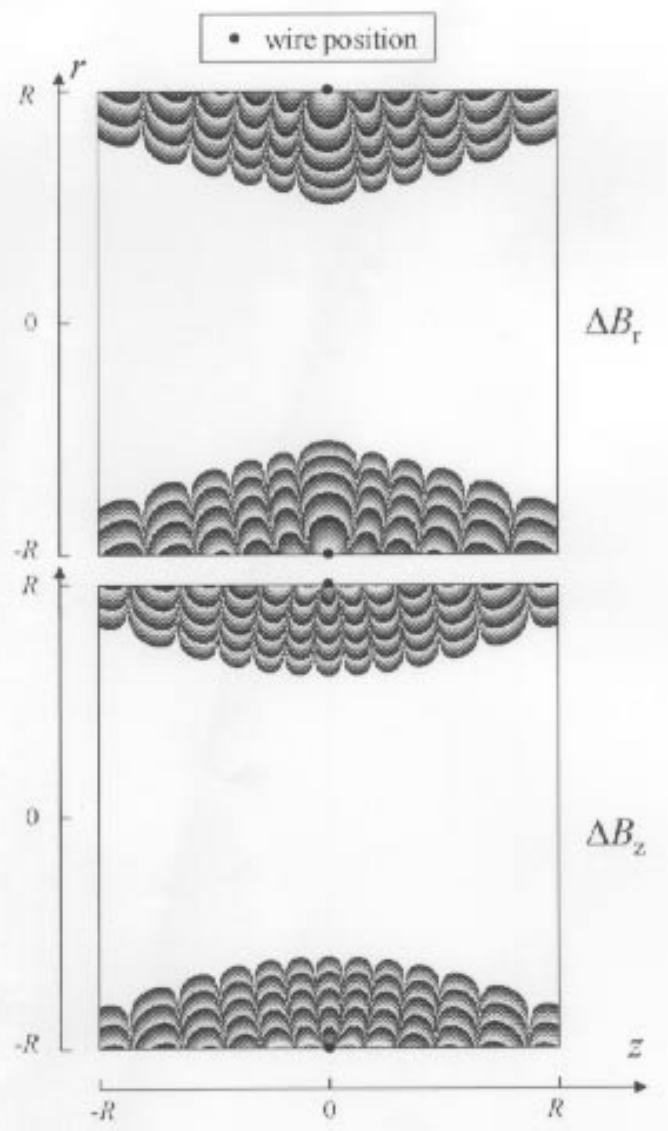

Figure 4: Contour maps of $\Delta B_{r, z}(r, z)$. (A) Radial component; (B) Axial component. Scale: logarithmic.

The contour plots in Fig. 4 are $2 R \times 2 R$ logarithmic maps of the relative differences $\Delta B_{z}(r, z)$ and $\Delta B_{r}(r, z)$, respectively, in the $(r, z)$-plane. In these 
figures the black-white transitions edges define contours where the magnetic field, calculated with the BS method and with the proposed method using $N=10$, present a constant difference according to $10^{k}=\Delta B_{\gamma}$, with $k=1, \cdots, 5$. Additionally a logarithmic grey scale was introduced in these figures to increase resolution, and the wire position is marked by solid circles. The extended white regions at the center of both figures, which represent differences in magnetic field that are less than $10 \mathrm{ppm}$, demonstrates that the vality region of this method, at $\mathbf{z}=\mathbf{0}$, is a disk of radius $\mathbf{R}_{\mathbf{d}} \leq \mathbf{0 . 6 R}$ but for $\mathbf{z} \neq \mathbf{0}$ we have disks with radius that increase with $\mathbf{z}$.

\section{Conclusion}

In this work we proposed an analytical method to calculate the magnetic field from the BS law. This was applied to get expressions for calculating the magnetic field components due to a circular current distributions. This components can be expressed as a series expansion that converges over the extended region. The accuracy of the method inside the convergence region depends on the number of terms considered in the series expansion.

\section{Acknowledgement}

This research was partially supported by FAPESP project 96/05437-0.

\section{References}

[1] S. Crozier and D.M. Doddrell, J.Mag.Reson. 127, 233 (1997).

[2] E. Thomas, Jr., G.E. Sasser, S.F. Knowlton, et al. Comp.Phys.Comm. 100, 31 (1997).

[3] De-man Wang, Guang-mei Wei, Hui-cai Xie, et al., IEEE Trans. on Magn. 31, 2228 (1995).

[4] D. Tomasi, E.C. Caparelli, H. Panepucci and B. Foerster, J.Mag.Reson. 140, 325 (1999).

[5] J.D. Jackson, Classical Electrodynamics, (Wiley, New York, 1975).

[6] K.Yoda, J. Appl Phys. 67, 4349 (1990).

[7] E.C. Caparelli, D. Tomasi and H. Panepucci, J.Mag.Reson.Imag. 9, 725 (1999).

[8] I.S. Gradshteyn and I.M. Ryzhik, Table of Integrals, Series, and Products, Academic Press, Inc. (1980). 